\title{
Thermoelectric Properties and their Stability of $( \pm)$-10-Camphorsulfonic Acid Doped Polyaniline Films on Flexible Substrate
}

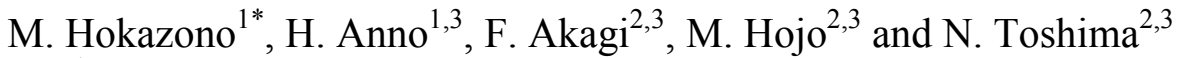 \\ ${ }^{1}$ Department of Electrical Engineering, Tokyo University of Science, Yamaguchi, \\ 1-1-1 Daigaku-dori, Sanyoonoda 756-0884, Japan \\ ${ }^{2}$ Department of Applied Chemistry, Tokyo University of Science, Yamaguchi, \\ 1-1-1 Daigaku-dori, Sanyoonoda 756-0884, Japan \\ ${ }^{3}$ Advanced Materials Institute, Tokyo University of Science, Yamaguchi, \\ 1-1-1 Daigaku-dori, Sanyoonoda 756-0884, Japan \\ *Fax: 81-836-88-4541, e-mail: masa2a@rs.tus.ac.jp
}

\begin{abstract}
We investigated the important properties for practical use of ( \pm )-10-camphorsulfonic acid (CSA) doped polyaniline (PANI) films on polyimide substrate as thermoelectric materials. We tested the flexibility and the thermal stability of electrical conductivity and Seebeck coefficient for the CSA-PANI films. The electrical conductivity and the Seebeck coefficient for the CSA-PANI films were almost unchanged after bending of 10,000 times. In addition, the CSA-PANI films were not exfoliated from the substrate after the bending test. The thermal stability of electrical conductivity and Seebeck coefficient for a CSA-PANI film on polyimide substrate was tested by the heat cycles of 30 times in the temperature range of 330-380 K. After the heat cycle test, the Seebeck coefficient slightly increased (approximately 10\%) while the electrical conductivity slightly decreased (approximately 5\%). The CSA-PANI film on polyimide substrate was found to be mechanically tough, flexible and possess relatively good thermal stability of electrical conductivity and Seebeck coefficient. These results suggest that if the thermoelectric properties are improved, CSA-PANI films are promising for use of the flexible organic thermoelectric devices. Key words: polyaniline film, thermoelectric properties, flexibility, thermal stability
\end{abstract}

\section{INTRODUCTION}

Conducting polymers, such as polyaniline (PANI) and polypyrrole, have several attractive features for use as thermoelectric materials because of the low thermal conductivity, which is one order of magnitude lower than inorganic thermoelectric materials, as well as their potential low cost due to available resources, easy synthesis, and easy processing into a versatile form $[1$, 2]. Recently, hybrid materials of organic and inorganic compounds have received much attention because of their unique properties and potential applications in a variety of areas [3]. In the thermoelectric field, the nanohybrid between conducting polymers and inorganic thermoelectric materials is an approach to realize new nanostructured materials with enhanced thermoelectric properties. We reported the thermoelectric properties of the ( \pm -10-camphorsulfonic acid (CSA) doped PANI/inorganic material composite films $[4,5]$.

Another approach to the improvement of thermoelectric figure of merit $Z T$ is the carrier tuning of CSA-PANI films. The electrical properties of PANI can be controlled both by charge transfer doping and by protonation. PANI is converted from the semiconducting (insulating) emeraldine base from to the conducting emeraldine salt form by doping with camphorsulfonic acid [6-8]. Anno et al. [9] reported that PANI films doped with different concentration of CSA with molar ratio $x$ of CSA to two phenylnitrogen units of $x=0.2$ to 1. The maximum thermoelectric figure of merit $Z T$ in the in-plane direction was reported to be $10^{-3}$ for $x=1$ by Anno et al. [9]. From the practical point of view, flexibility, thermal stability of thermoelectric properties, and durability are important, but there are few studies of these properties for CSA-PANI films as thermoelectric materials. In this study, we investigated the important properties, flexibility and thermal stability, of CSA-PANI (CSA molar ratio $x=1$ ) films on polyimide substrate as thermoelectric materials.

\section{EXPERIMENTAL PROCEDURE}

The emeraldine base form of dedoped PANI with high molecular weight $\left(M_{\mathrm{w}} \sim 80,000\right)$ was chemically prepared according to the procedure previously reported by Adams et al. [10]. CSA-PANI samples were prepared with molar ratio of a CSA to tow phenyl-nitrogen units. The dedoped PANI was mixed with CSA in an agate mortar and pestle. The CSA-PANI mixture was then dissolved into an appropriate quantity of $m$-cresol (weight ratio of PANI: $m$-cresol $=1: 100$ ). The solution was sonicated for $2 \mathrm{~h}$ for dispersion. The CSA-PANI films were prepared by casting of the dispersed solution onto a flexible polyimide substrate and subsequently drying slowly in air on a hot plate at $313 \mathrm{~K}$ for a few days. The product was then dried in a vacuum at $313 \mathrm{~K}$ for $12 \mathrm{~h}$, and most of the $m$-cresol was removed.

The Seebeck coefficient and the electrical conductivity were measured simultaneously in the temperature range from $\sim 310$ to $\sim 400 \mathrm{~K}$ by using a thin film measurement system (ULVAC ZEM 3-M8) or a home-made system. In the thin film measurement system, the measurement errors of the Seebeck coefficient and the electrical conductivity for a standard sample (constantan) were less than $\pm 7 \%$ and $\pm 10 \%$, respectively. The values of Seebeck coefficient and 
electrical conductivity agreed well between the two systems. For both systems, a standard four-probe method with a direct-current (DC) power supply of $0.05-0.10 \mathrm{~mA}$ was used for the electrical conductivity measurements. The Seebeck coefficient was obtained from the thermo-electromotive force versus temperature difference within $10 \mathrm{~K}$. The absolute Seebeck coefficient was obtained by subtracting the Seebeck coefficient of the platinum probe wire over the entire temperature range. We tested the flexibility and the thermal stability of electrical conductivity and Seebeck coefficient for the CSA-PANI films on polyimide substrate. The flexibility of a CSA-PANI film on polyimide substrate was tested by repeating the bending over a radius of curvature (10 $\mathrm{mm}) 10,000$ times, see Fig. 1. To examine the thermal stability, the electrical conductivity and Seebeck coefficient for a CSA-PANI film on polyimide substrate was measured during heating and cooling cycles of 30 times in the temperature range of $330-380 \mathrm{~K}$.

\section{RESULTS AND DISCUSSION}

Figure 2 shows the temperature dependence of Seebeck coefficient and electrical conductivity for a CSA-PANI film on polyimide substrate. The values of Seebeck coefficient and electrical conductivity of the CSA-PANI film are about $10.0 \mu \mathrm{V} / \mathrm{K}$ and about 190 $\mathrm{S} / \mathrm{cm}$, respectively, near room temperature. The electrical conductivity value is comparable to the typical room temperature values reported by Reghu at al. [6-8] for CSA-PANI films on glass substrate (about $200 \mathrm{~S} / \mathrm{cm}$ to about $400 \mathrm{~S} / \mathrm{cm}$ ). The maximum value of thermoelectric power factor $\mathrm{PF}=S^{2} \sigma$, where $S$ is the Seebeck coefficient and $\sigma$ is the electrical conductivity, of the CSA-PANI film on the polyimide substrate is 2.5 $\mu \mathrm{Wm}^{-1} \mathrm{~K}^{-2}$ at $380 \mathrm{~K}$. It is noted that the value of PF for the CSA-PANI film on polyimide substrate is comparable to the maximum value of PF reported by Anno et al. [9]. The high value of PF for CSA-PANI film on polyimide substrate is due to the relatively high electrical conductivity (about $190 \mathrm{~S} / \mathrm{cm}$ ).

Figure 3 shows the temperature dependence of Seebeck coefficient $S$ (a) and electrical conductivity $\sigma$ (b) for a CSA-PANI film on polyimide substrate before and after bending of 10,000 times. The Seebeck coefficient and the electrical conductivity for the CSA-PANI film on polyimide substrate were almost unchanged after bending of 10,000 times. In addition, the CSA-PANI film on polyimide substrate was not exfoliated from the polyimide substrate after bending of 10,000 times. It was found that CSA-PANI films on polyimide substrate possessed both mechanical toughness and flexibility. These features are advantages to use CSA-PANI for flexible thermoelectric devices.

Figure 4 shows the Seebeck coefficient and the electrical conductivity at $380 \mathrm{~K}$ as a function of heat cycles for a CSA-PANI film on polyimide substrate. As the number of heat cycles increases, the Seebeck coefficient gradually increases while the electrical conductivity gradually decreases. It was found that the CSA-PANI film on polyimide substrate possessed relatively good thermal stability on the electrical conductivity and the Seebeck coefficient. The slight changes in the thermoelectric properties are attributed to the decrease in the carrier concentration of the

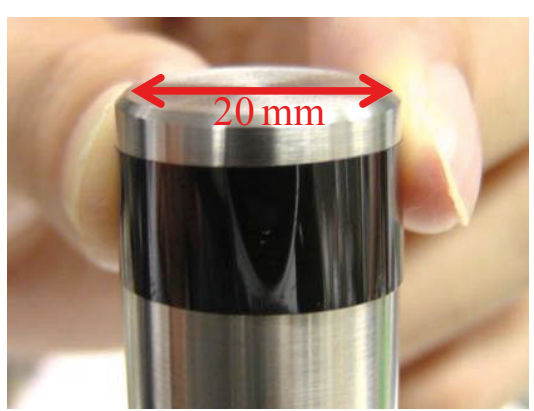

Fig. 1. Bending test for CSA-PANI film on polyimide substrate.

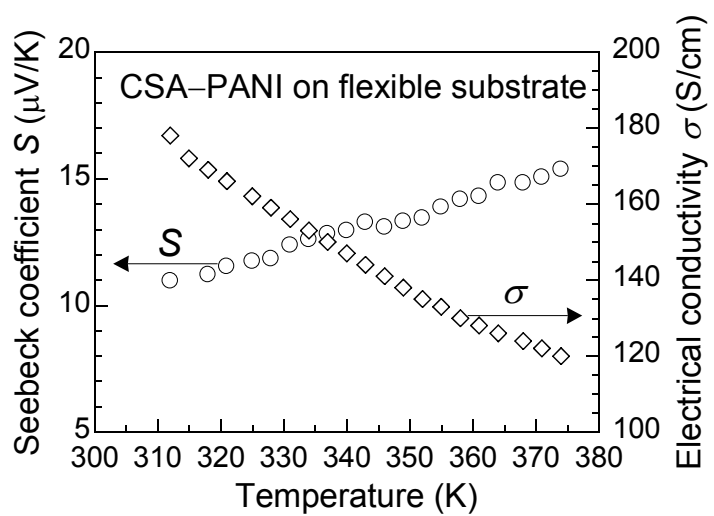

Fig. 2. Temperature dependence of Seebeck coefficient and electrical conductivity for a CSA-PANI film on polyimide substrate.

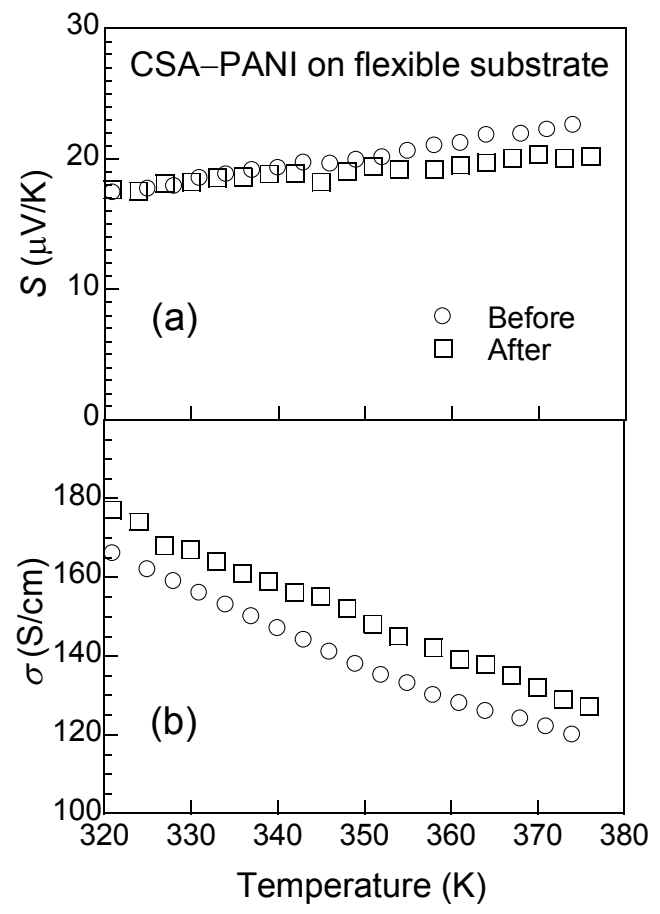

Fig. 3. Temperature dependence of Seebeck coefficient $S$ (a) and electrical conductivity $\sigma$ (b) for a CSA-PANI film on polyimide substrate before and after bending of 10,000 times. 


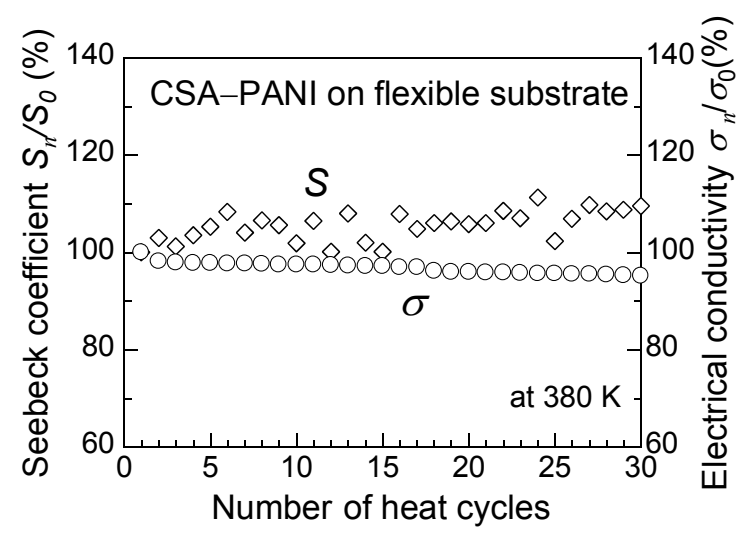

Fig. 4. Seebeck coefficient and electrical conductivity at $380 \mathrm{~K}$ as a function of heat cycles for a CSA-PANI film on polyimide substrate.

CSA-PANI film due to the evaporation of $m$-cresol or CSA in heat cycles between 330 and $380 \mathrm{~K}$. Generally, the devices of organic materials are sealed in order to protect the oxidation by air or decomposition. The changes in the Seebeck coefficient and the electrical conductivity for the CSA-PANI film on polyimide substrate by heat cycles may be solved by sealing techniques.

\section{CONCLUSION}

We have investigated the flexibility and thermal stability, which is important properties for practical use, of CSA-PANI films on the polyimide substrate as thermoelectric materials. As a result of bending test (10,000 times), it was found that CSA-PANI films on the polyimide substrate possessed good mechanical toughness and flexibility. The values of Seebeck coefficient and the electrical conductivity for CSA-PANI film on polyimide substrate were almost unchanged after heating and cooling cycles of 30 times. These results show that if the thermoelectric properties of CSA-PANI films are significantly improved, the CSA-PANI film is very promising for use of flexible thermoelectric devices.

\section{ACKNOWLEDGMENTS}

This work is supported by MEXT Regional Innovation Strategy Support Program (Global Type) Yamaguchi Green Valley and JSPS KAKENHI Grant Number 21310077.

\section{REFERENCES}

[1] H. Yan, and N. Toshima, Chem. Lett., 28, 1217 (1999).

[2] N. Toshima, H. Yan, and M. Kajita, IEEE Proc. XXI Int. Conf. on Thermoelectrics, (Long Beach, CA, USA, 25-29 August 2002), 147 (2002).

[3] N. Toshima, Macromol. Symp., 235, 1 (2006).

[4] H. Anno, M. Fukamoto, Y. Heta, K. Koga, H. Itahara, R. Asahi, R. Satomura, M. Sannomiya, and N. Toshima, J. Electron. Mater., 38, 14431449 (2009).

[5] H. Anno, K. Yamaguchi, T. Nakabayashi, H. Kurokawa, F. Akagi, M. Hojo, and N. Toshima, IOP Conf. Ser.: Mater. Sci. Eng., 18, 142003 (2011).

[6] M. Reghu, Y. Cao, D. Moses, and A.J. Heeger, Phys. Rev. B, 47, 1758 (1993).

[7] M. Reghu, C.O. Yoon, D. Moses, A.J. Heeger, and Y. Cao, Phys. Rev. B, 48, 17685 (1993).

[8] C.O. Yoon, M. Reghu, D. Moses, Y. Cao, and A.J. Heeger, Synth. Met., 69, 273 (1995).

[9] H. Anno, M. Hokazono, F. Akagi, M. Hojo, and N. Toshima, J. Electron. Mater., DOI:10.1007/ s11664-012-2368-z, (2012).

[10] P.N. Adams, P.J. Laughlin, A.P. Monkman, and A.M. Kenwright, Polymer, 37, 3411 (1996).

(Received March 4, 2013; Accepted April 6, 2013) 